\title{
The Application Research of Cellular Genetic Algorithm in Vehicle Routing Problem
}

\author{
Youzhi Jin ${ }^{1,2}$ \\ School of Computer Science and Engineering, Tianjin University of Technology \\ Tianjin, 300384 ,China \\ E-mail:jin_you_zhi@qq.com \\ Jianhua Fan ${ }^{3}$ \\ School of Computer Science and Engineering, Tianjin University of Technology \\ Tianjin, 300384 ,China \\ E-mail:fan109@163.com
}

Jie Yang ${ }^{\mathrm{a}}$; Jiawei Li ${ }^{\mathrm{b}}$; Deliang Fan ${ }^{\mathrm{c}}$

School of Computer Science and Engineering, Tianjin University of Technology

Tianjin, 300384 ,China

E-mail: ${ }^{a} 948100998$ Qqq.com; ${ }^{b} 1740459051$ Qqq.com; 'cfan0819fandfoxmail.com

\begin{abstract}
In order to solve the Distance-constrained Capacitated Vehicle Routing Problem ( Distanceconstrained CVRP), the cellular genetic algorithm(CGA) is used in this paper. A new crossover operator--SAX which can better reflect the self-adaptability of CGA is proposed, and three types of neighborhood are introduced to analyse the search performance of CGA in Distanceconstrained CVRP. Two instances are introduced to show the feasibility of CGA in solving Distance-constrained CVRP. The experimental results show that the performance of CGA is obviously better than that of the traditional genetic algorithm, and the optimization results of the vehicle routings are improved. Especially, the Moore neighbor structure indicates better search efficiency for SAX crossover operator. Because of the strong searching ability, CGA can effectively solve the optimization of vehicle routing problems.
\end{abstract}

ISCC2017

16-17 December 2017

Guangzhou, China

\footnotetext{
${ }^{1}$ Speaker

${ }^{2}$ This study is supported by Innovative Training Project for College Students

(No.201610060073)

${ }^{3}$ Corressponding Author
}

(C) Copyright owned by the author(s) under the terms of the Creative Commons 


\section{Introduction}

In the 1950's, Von Neumann, the father of modern computer theory, put forward the concept of Cellular Automaton(CA), and constructed a machine that can replicate itself. Since 1990's, the application of CA has been developing rapidly in many fields. In recent years, some scholars have begun to study the combination of CA and intelligent optimization algorithms. In 1993, Whitel first proposed the concept of cellular genetic algorithm(CGA), and then Tomassini et al conducted continuous study of CGA in many aspects[1-3]. CGA is a kind of genetic algorithm(GA) in which the population is structured in a specified topology so that individuals may only interact with their neighbors. In the past 10 years, the research in this field has been gradually active. The combination of CA structural features and GA can simulate not only the biological evolution process, but also the living environment of organisms. Some studies have shown that compared with the traditional GA, the CGA has better performance in solving complex and multimodal optimization problems.

The Vehicle Routing Problem(VRP) consists in delivering goods to a set of customers with known demands through minimum cost vehicle routes, beginning and finishing at the depot. The VRP has been the subject of widespread concern since Dantzig and Ramzer proposed it in 1959. The VRP is a NP-hard problem and has many industrial applications, being studied both theoretically and practically, resulting in significant reduction of total cost. Due to the practical relevance of VRP and its NP-hardness, many heuristic or meta-heuristic solution methods have been proposed to solve the VRP. Some examples include Tabu Search[4], Genetic Algorithm[5], Simulated Annealing[6], Ant Colony[7] and Particle Swarm[8] and so on. These methods have the potential to jump out of the local optima and wide adaptability, and become the main method to study the VRP.

CGA has been successfully used in solving many optimization problems, but the application research in the VRP is still in the initial stage. In 2004, Alba first solved a large benchmark of the Capacitated Vehicle Routing Problem(CVRP) with a CGA in which each individual could only interact with its neighbors to ensure the diversity of population and improve search efficiency. The local optimization step by applying 2-Opt and $\lambda$-Interchange was used to get the best known results[9]. Then, in 2008, Alba further perfected his work, and proposed a hybrid cellular genetic algorithm[10]. In 2010, Kamkar firstly used CGA to solve the Vehicle Routing Problem with Time Window(VRPTW), and the simulation experiment data showed that CGA had superior search performance compared with other heuristic algorithms[11]. D L Zhu and Y M Qin studied the VRP for express delivery based on automatic parcel machine and the VRPTW by using CGA respectively[12,13].

In this paper, we consider the Distance-constrained Capacitated Vehicle Routing Problem (Distance-constrained CVRP) in whih a fixed fleet of vehicles must serve customers from a common depot at minimum travel costs. The customer demands for a single commodity are known, and vehilce capacity is the same. Each route must start and end at the depot, and each customer must be served exactly once by one vehicle. The total demand of any route does not exceed the vehicle capacity and the distance of any route does not exceed an upper limit.

Although the VRP has been studied by CGA in some literature, few works have addressed the influence of different neighbor forms on the search results of vehicle routing and the selfadaptability of the CGA.

\section{Cellular Genetic Algorithm(CGA)}




\subsection{Neighborhood}

In CGA, cells distribute in two-dimensional grid of size $n \times n$, and cells represent individuals in population. The most frequently used neighborhoods are Von Neumann, Moore and Extended Moore, so each cell can have 4, 8 or 24 neighbors, as shown in Figure1 (a) - (c).

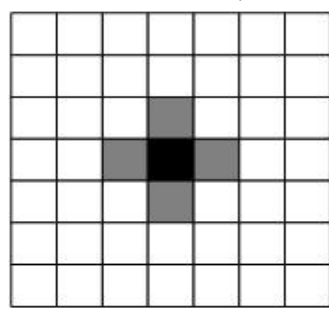

(a) Von Neumann



(b) Moore



(c) Extended Moore

Figure 1 :The Neighborhood Type

\subsection{The Steps of CGA}

(1) Population initialization: In the two-dimensional grid of size $n \times n$, each cell represents an individual $V_{i, j}(i, j=1,2, \ldots, n)$ which has a chromosome $x_{i, j}$, whose coordination is $i$ and $j$.

(2) Encoding: Similar to the genetic algorithm, the appropriate encoding method is adopted according to the different problems.

(3) Computation of fitness: The fitness of individual $V_{i, j}$ is calculated and denoted by $\operatorname{eval}\left(V_{i, j}\right)$ 。

(4) Selection: Each central cell $V_{i, j}$ performs selection operations among the neighbors whose fitness is greater than or equal to itself $\left(\operatorname{eval}\left(V_{a, b}\right) \geq \operatorname{eval}\left(V_{i, j}\right)\right)$ according to certain strategy.

(5) Crossover: Unlike the traditional GA, here the central cell $V_{i, j}$ and the selected neighbor cell $V_{a, b}$ performs crossover operation according to some manner.

(6) Mutation: Similar to the GA, the mutation operation is carried out between the center cell and neighbor cell after crossover.

(7) Determine whether the end condition of the algorithm is reached, if not to step(3).

(8) Output the current optimal solution.

\section{The CGA for Distance- constrained CVRP}

\subsection{Representation of Individuals}

The initial population consisting of permutations of natural numbers is randomly generated. Each permutation only contains customers, so we will use a permutation of numbers[1...n] with length $n$ for representing a solution for the VRP with $n$ customers. The number of vehicles needed is determined through calculation. For example, the permutation for an individual is 137856429 , which indicates arranging vehicles for customers according to the order of $1 \rightarrow 3 \rightarrow 7 \rightarrow 8 \rightarrow 5 \rightarrow 6 \rightarrow 4 \rightarrow 2 \rightarrow 9$. Vehicle 1 begins at the depot, visits customer 1 , and continues the service for customer 3 if the remaining capacity can satisfy it. Otherwise, vehicle 1 returns to depot, and vehicle 2 is rearranged to serve customer 3 and so on. 
This permutation manner need to determine each route by calculation. It is possible that the number of vehicles needed is less than the number of vehicles available, and the permutation method is convenient for the crossover and mutation operations.

\subsection{Fitness Value}

If the routes corresponding to the individual $V_{i, j}$ do not exceed the number of vehicles available and traveling distance limit, the total costs $z\left(V_{i, j}\right)$ of $V_{i, j}$ are the sum of the length of all routes. Otherwise, $z\left(V_{i, j}\right)$ is computed by the sum of the length of all routes plus the number of vehicles exceeding multiplied by the penalty coefficient $(P w)$. Since the objective of the VRP is to minimize total costs, the fitness function can be expressed as eval $\left(V_{i, j}\right)=1 / z\left(V_{i, j}\right)$.

\subsection{Selection Operation}

The three neighborhoods mentioned above are used in the selection operation. For each central cell $V_{i, j}$, the ones whose fitness is greater than or equal to it's own (represented by set $\Omega$ ) are selected from the neighbors(including itself) $\left(\operatorname{eval}\left(V_{a, b}\right) \geq \operatorname{eval}\left(V_{i, j}\right)\right)$. For each cell $V_{m, n}$ in set $\Omega$, the fitness evaluation value is computed as follows .

$$
p_{m, n}=\frac{\operatorname{eval}\left(V_{m, n}\right)}{\sum_{a, b \in \Omega} \operatorname{eval}\left(V_{a, b}\right)}
$$

The $p_{m, n}$ represents the probability of the $V_{m, n}$ being selected. All the cells in set $\Omega$ are arranged in a certain order and the cumulative probability $q_{m, n}$ for each cell $V_{m, n}$ is calculated. Unlike the GA, the selection operation is done by Roulette among individuals in set $\Omega$, which is equivalent to the process of central cell learning from neighbors. A random number $r$ is produced in the interval [0,1], and the individual is chosen according to $r$ and cumulative probability of each cell. The wheel turns once, and the selected individual is the learning object of central cell $V_{i, j}$.

\subsection{Crossover Operator}

In this paper we use two kinds of crossover operator. One is the order reverse crossover(ORX)[12], the other is the self-adaptability crossover proposed in this paper which determines the learning content of the central cell according to the degree of excellence of neighborhood individual. If the neighbor cell $V_{a, b}$ is much better than the central cell $V_{i, j}$, the individual $V_{i, j}$ will learn more from the neighbor $V_{a, b}$, otherwise it will be less learned. Supposing the crossover probability is $p_{c}$, the probability values $p$ for central cell $V_{i, j}$ is calculated as follows.

$$
p=p_{c}+\left(1-p_{c}\right) \frac{\operatorname{eval}\left(V_{a, b}\right)-\operatorname{eval}\left(V_{i, j}\right)}{\operatorname{eval}\left(V_{a, b}\right)-\operatorname{eval}\left(V_{i, j}\right) p_{c}}
$$

The probability $p$ represents how much the central cell $V_{i, j}$ learn from neighbor $V_{a, b}$. The length of the cross segment is determined by the probability $p$ and permutation length, then the ORX crossover operator is used between individual $V_{i, j}$ and $V_{a, b}$, and the central cell $V_{i, j}$ is replaced. Probability $p$ can be regarded as the degree of self confidence or learning tendency of 
individual $V_{i, j}$, and it can also guarantee the generation of new individuals when selecting their own to cross.

\subsection{Mutation Operator}

The inversion mutation operator is used which selects a section of permutation [ $\operatorname{ran} 1$, ran2] randomly and reverses it(see Figure 2).

\begin{tabular}{|c|c|c|c|c|c|c|c|c|}
\hline \multicolumn{9}{|c|}{ Before mutation: } \\
\hline 1 & 2 & 3 & 4 & 5 & 6 & 7 & 8 & 9 \\
\hline \multicolumn{9}{|c|}{ After mutation: } \\
\hline 1 & 2 & 6 & 5 & 4 & 3 & 7 & 8 & 9 \\
\hline
\end{tabular}

Figure 2: Inversion Mutation Operator

\section{Experimentation}

In the Visual Studio 2015 environment, the experimental simulation analysis software is written in C\# language. The Distance-constrained CVRP is implemented by using GA, CGA with Von Neumann, Moore and Extended Moore neighborhoods. They have been tested with instances in literature[14] and literature[15].

\subsection{GA vs CGA}

We will compare the behavior of GA and CGA with instances in this section, in which the maximum mileage per vehicle is not more than 50 kilometers. The iterations of algorithms is set to 1000 , crossover probability is 0.6 to 0.8 and mutation probability is 0.1 . The order reverse crossover(ORX) is used as crossover operator. In order to meet the requirements of the twodimensional space of CGA, the population size is set to 144, 100 and 64 respectively. Each algorithm runs 10 times and the average total traveling length of the solutions found is shown in Table 1 and Table 2.

\begin{tabular}{|c|c|c|c|c|c|}
\hline $\begin{array}{c}\text { Crossover } \\
\text { probability }\end{array}$ & $\begin{array}{c}\text { Population } \\
\text { size }\end{array}$ & GA & $\begin{array}{c}\text { CGA- } \\
\text { Von }\end{array}$ & CGA-M & $\begin{array}{c}\text { CGA- } \\
\text { EM }\end{array}$ \\
\hline \multirow{4}{*}{0.6} & 144 & 68.4 & 67.5 & 67.5 & 67.5 \\
\cline { 2 - 6 } & 100 & 68.2 & 67.5 & 67.5 & 67.5 \\
\cline { 2 - 6 } & 64 & 68.3 & 67.5 & 67.5 & 67.5 \\
\hline \multirow{4}{*}{0.7} & 144 & 68 & 67.5 & 67.5 & 67.5 \\
\cline { 2 - 6 } & 100 & 68.5 & 67.5 & 67.5 & 67.5 \\
\cline { 2 - 6 } & 64 & 68.4 & 67.5 & 67.5 & 67.5 \\
\hline \multirow{4}{*}{0.8} & 144 & 68.3 & 67.5 & 67.5 & 67.5 \\
\cline { 2 - 6 } & 100 & 68.8 & 67.5 & 67.5 & 67.5 \\
\cline { 2 - 6 } & 64 & 68.7 & 67.5 & 67.5 & 67.8 \\
\hline
\end{tabular}

Table 1: GA vs CGA for Instance in Literature[14]

\begin{tabular}{|c|c|c|c|c|c|}
\hline $\begin{array}{c}\text { Crossover } \\
\text { probability }\end{array}$ & $\begin{array}{c}\text { Population } \\
\text { size }\end{array}$ & GA & $\begin{array}{c}\text { CGA- } \\
\text { Von }\end{array}$ & CGA-M & $\begin{array}{c}\text { CGA- } \\
\text { EM }\end{array}$ \\
\hline
\end{tabular}




\begin{tabular}{|c|c|c|c|c|c|}
\hline \multirow{4}{*}{0.6} & 144 & 119.6 & 109.9 & 110.4 & 112.1 \\
\cline { 2 - 6 } & 100 & 117.2 & 111.4 & 112.4 & 113.8 \\
\cline { 2 - 6 } & 64 & 116.5 & 112.3 & 112.7 & 118.1 \\
\hline \multirow{4}{*}{0.7} & 144 & 121.2 & 110.1 & 111.3 & 113.7 \\
\cline { 2 - 6 } & 100 & 117.3 & 110.8 & 111.7 & 115.2 \\
\cline { 2 - 6 } & 64 & 120.9 & 110.9 & 114.8 & 116.9 \\
\hline \multirow{3}{*}{0.8} & 144 & 117.3 & 109.8 & 110.6 & 112 \\
\cline { 2 - 6 } & 100 & 117.5 & 110.7 & 111.7 & 115.1 \\
\cline { 2 - 6 } & 64 & 120.9 & 110.8 & 112.9 & 118.1 \\
\hline
\end{tabular}

Table 2: GA vs CGA for Instance in Literature[15]

CGA-Von, CGA-M and CGA-EM mean CGA with Von Neumann, Moore and Extended Moore neighborhood respectively. The total traveling distance of optimal path in literature[14] and literature[15] is 67.5 and 107.8. As can be seen, the results of CGA are better than those of GA, and the neighborhood of Von Neumann type has better search performance than the other two neighbor structures.

In terms of time complexity, CGA need more computation time than GA in each evolution generation, which is mainly reflected in the selection process. This is especially true for the Extended Moore neighborhood. In terms of search performance and computation time, the Von Neumann and Moore neighbors are preferable. But the search speed of CGA is faster than GA, because the CGA can find the optimal solution more quickly with less evolution generation to meet the requirements.

\subsection{The Comparison of Crossover Operators}

In this section, we will study the behavior of self-adaptation crossover(SAX) proposed in this paper, and compare the results of SAX with those of ORX. Similarly, crossover probability is set to 0.6 to 0.8 , mutation probability is 0.1 and the population size is 144,100 and 64 respectively. CGA with three types of neighborhood are used, and each algorithm runs 10 times for each crossover operator under different crossover probabilities. The best results for ORX and SAX are shown in the Table 3.

\begin{tabular}{|c|c|c|c|c|}
\hline \multirow{2}{*}{ Algorithm } & \multirow{2}{*}{$\begin{array}{c}\text { Crossover } \\
\text { operator }\end{array}$} & \multicolumn{3}{|c|}{ Population size } \\
\cline { 3 - 5 } & & 144 & 100 & 64 \\
\hline \multirow{2}{*}{ CGA-Von } & ORX & 109.8 & 110.7 & 110.8 \\
\cline { 2 - 5 } & SAX & 109.7 & 110.3 & 110.2 \\
\hline \multirow{2}{*}{ CGA-M } & ORX & 110.4 & 111.7 & 112.7 \\
\cline { 2 - 5 } & SAX & 109.5 & 109.8 & 111.5 \\
\hline \multirow{2}{*}{ CGA-EM } & ORX & 112 & 113.8 & 116.9 \\
\cline { 2 - 5 } & SAX & 111.7 & 110.5 & 114.5 \\
\hline
\end{tabular}

Table 3: Comparison of Crossover Operators

As can be seen, the best results of $\operatorname{SAX}\left(p_{c}=0.6\right)$ are better than ORX, and the Moore neighbor structure in SAX operator has outstanding performance.

\section{Conclusion and Further Work}


This paper studies the Distance- constrained CVRP using CGA, and compares the results with GA. The search performance of three kinds of neighborhood is analyzed, and a selfadaptation crossover operator(SAX) is presented. The research results show that CGA has better search performance. Hence, for a future work, one can think about applying the CGA in the study of other types of vehicle routing problems.

\section{References}

[1] M Tomassini. Spatially structured evolutionary algorithms [M]. Springer, 2005.

[2] A Villagra, G Leguizamon, E Alba. Active components of metaheuristics in cellular genetic algorithms[J]. Soft Computing, Methodologies and Applications, 2015, 19(5): 1295-1309.

[3] A N Asmaa, T E Ahmet, A Tughrul. Adaptive three-dimensional cellular genetic algorithm for balancing exploration and exploitation processes[J]. Springer-Verlag, 2013, 17(7): 1145-1157.

[4] M Bolduc, G Laporte, J Renaud. A tabu search heuristic for the split delivery vehicle routing problem with production and demand calendars $[\mathrm{J}]$. European Journal of Operational Research, 2010, 202(1): 122-130.

[5] A E Amraoui, M A Manier, A E Moudni, M Berrejeb. A genetic algorithm approach for a single hoist scheduling problem with time windows constraints [J]. Engineering Applications of Artificial Intelligence, 2013, 26(7): 1761-1771.

[6] D Mu, C S Wang, S C Wang, S C Zhou. Solving TDVRP based on parallel-simulated annealing algorithm $[\mathrm{J}]$. Computer Integrated Manufacturing Systems, 2015, 21(6): 1626-1636.(InChinese)

[7] S R Balseiro, I Loiseau, J Ramonet. An ant colony algorithm hybridized with insertion heuristics for the time dependent vehicle routing problem with time windows [J]. Computers \& Operations Research, 2011, 38(6): 954-966.

[8] B F Moghaddam, R Ruiz, S J Sadjadi. Vehicle routing problem with uncertain demands: an advanced particle swarm algorithm [J]. Computers \& Industrial Engineering, 2012, 62(1): 306-317.

[9] E Alba, B Dorronsoro. Solving the vehicle routing problem by using cellular genetic algorithms [J]. Lecture Notes in Computer Science, 2004, 3004: 11-21.

[10] E Alba, B Dorronsoro. A hybrid cellular genetic algorithm for the capacitated vehicle routing problem[J]. Springer Berlin Heidelberg, 2008, 82:379-422.

[11] I Kamkar, M Poostchi, M Totonchi. A cellular genetic algorithm for solving the vehicle routing problem with time windows [J]. Soft Computing in Industrial Applications, 2010, 75:263-270.

[12] D L Zhu, T Zhan, Y Zhang, Z Liu. Logistics distribution route optimization based on cellular niche genetic algorithm[J]. Modular Machine Tool \& Automatic Manufacturing Technique, 2013(1): 121125.(InChinese)

[13] Y M Qin, H J Mao, X L Hei. Optimizing of vehicle routing for express delivery based on automatic parcel machine $[\mathrm{J}]$. Journal of Highway and Transportation Research and Development, 2015, 32(10): 134-140.(InChinese)

[14] Q X Feng, C Liu, Z Jia. Improved genetic algorithms for vehicle routing problem $[\mathrm{J}]$. MATHEMATICS IN PRACTICE AND THEORY, 2008, 38(13): 123-128.(InChinese)

[15] M X Lang, S J Hu. Study on the optimization of physical distribution routing problem by using hybrid genetic algorithm[J]. Chinese Journal of Management Science, 2002, 10(2): 51-56. (InChinese) 